\title{
Theoretical limits of the multistacked 1-D and 2-D microstructured inorganic solar cells
}

\author{
Emre Yengel*a, Hakan Karaagac ${ }^{\mathrm{b}}$, Logeeswaran $\mathrm{VJ}^{\mathrm{c}}$, M. Saif Islam ${ }^{\mathrm{c}}$ \\ ${ }^{a}$ National Nanotechnology Research Center, Bilkent University UNAM, Bilkent, Ankara TURKEY \\ 06800; ${ }^{b}$ Dept. of Physics Eng., Istanbul Technical University, Maslak, Istanbul TURKEY; ${ }^{\mathrm{c} D e p t . ~ o f ~}$ \\ Electrical and Computer Eng., University of California-Davis, CA USA 95616.
}

\begin{abstract}
Recent studies in monocrystalline semiconductor solar cells are focused on mechanically stacking multiple cells from different materials to increase the power conversion efficiency. Although, the results show promising increase in the device performance, the cost remains as the main drawback. In this study, we calculated the theoretical limits of multistacked 1D and 2D microstructered inorganic monocrstalline solar cells. This system is studied for $\mathrm{Si}$ and $\mathrm{Ge}$ material pair. The results show promising improvements in the surface reflection due to enhanced light trapping caused by photon-microstructures interactions. The theoretical results are also supported with surface reflection and angular dependent power conversion efficiency measurements of 2D axial microwall solar cells. We address the challenge of cost reduction by proposing to use our recently reported mass-manufacturable fracture-transfer- printing method which enables the use of a monocrystalline substrate wafer for repeated fabrication of devices by consuming only few microns of materials in each layer of devices. We calculated thickness dependent power conversion efficiencies of multistacked $\mathrm{Si} / \mathrm{Ge}$ microstructured solar cells and found the power conversion efficiency to saturate at $\% 26$ with a combined device thickness of $30 \mu \mathrm{m}$. Besides having benefits of fabricating low-cost, light weight, flexible, semi-transparent, and highly efficient devices, the proposed fabrication method is applicable for other III-V materials and compounds to further increase the power conversion efficiency above $35 \%$ range.
\end{abstract}

Keywords: Si/Ge solar cell, multistacked PV, fracture-transfer-printing, 1-D and 2-D microstructure

\section{INTRODUCTION}

Nowadays, the manufacturing of high-efficiency solar cells from inorganic monocrystalline semiconductors have generally been limited to their high cost. In order to produce low-cost single crystal based solar cells a variety of solutions and models have been proposed so far. Thin film based solar cells are among the most promising candidates and have been studied extensively so far. They offer a wide variety of opportunities compared with single crystalline based solar cells, such as using very small amount of material, large area process and deposition onto flexible substrate materials. Although thin film solar cells have raised the expectation of being alternative to single crystal based solar cell, the global production volume of them is still very low and more research is necessary for commercial thin film photovoltaic technology. Organic solar cells are other alternative candidates to solve the cost problem of solar cells but they are still constrained by their inherent problems, such as lack of long term stability, low efficiency (5-9\%) and poor homogeneity compared to their rigid, monocrystalline counterparts. There are also single crystal based novel approaches for the production of high efficient solar cells. Of these, the most important approach is the design of multi-junction solar cells consisted of several single junction solar cells (subcells). In such a design subcells with different bandgap energies are stacked upon each other to more efficiently capture a wider wavelength range of solar spectrum that leads to improvement in conversion efficiency over the single-junction solar cell. In monolithic multi junction (MJ) solar cells different materials with similar crystal structure are grown directly on top of each other using the same substrate. The requirement of similar structure of materials of each subcell limits the usage of different semiconductors. The most important difficulty associated with monolithic MJ design comes from the current-matching constraint owing to the series connection of different cells. The extracted current from a monolithic MJ solar cell is limited to the smallest value of current supplied by any of the individual subcell in the array. In addition, another constraint associated with monolithic design is that they are designed such that currents are matched under standard test conditions, but they tend to deviate under field conditions, which result in destruction current matching balance between each subcell. The transportation of generated current between subcells in monolithic design is achieved by the embedded tunnel junctions between subcells. In general, monolithic MJ solar cells become practical with light concentrators under illumination of at

Thin Films for Solar and Energy Technology VII, edited by Louay A. Eldada, Michael J. Heben, Proc. of SPIE Vol. 9561, $956103 \cdot$ ? 2015 SPIE $\cdot$ CCC code: 0277-786X/15/\$18 · doi: 10.1117/12.2188355 
least 400 suns. Currently, the most efficient monolithic MJ solar cells are made of GaInP, GaAs, and Ge layers on Ge substrate[1]. In monolithic multi-junction solar cells, the overall efficiency in several material combinations was experimentally demonstrated to be above $30 \%$ for two junctions and above $35 \%$ to $40 \%$ for three or more junctions.

Mechanically stacking of subcells is a promising alternative of monolithic MJ solar cells and is a potential candidate design for solving inherent problems associated with monolithic structure. The constraints of current and lattice matching, tunnel diodes and light concentrators can be avoided by such a design, which allows the extraction of power independently from connected individual subcells in MJ solar cell. In addition, mechanical stacking with distinct electrical contacts to each layer facilities the optimization of solar power absorption and guards against the uncertainty and homogeneity in the solar cell illumination spectrum during day, which means solving the one of the major problem associated with monolithic MJ solar cells.

In general, in commercial solar cells, wafer used for fabricating highly efficient solar cells, less than $2 \%$ material is used for designing active devices while the rest of the wafer materials offer only mechanical support. That means the photon absorption layer is typically $<10 \%$ of substrate thickness $(200-350 \mu \mathrm{m})$ while the remainder of the substrate functions as a mechanical support during manufacturing and installation processes. There have been a few notable transformative research demonstrations on heterogeneous integration of monocrystalline semiconductor materials for applications in solar cells using techniques like epitaxial lift-off $[2,3]$ wafer bonding [4-6] and heteroepitaxy[7-11]. These methods allow for some high-performance devices, but increase their cost-to-performance ratio (CPR) due to formidable technological challenges and limitations such as process incompatibilities from high growth temperature, high substrate cost, lack of substrate flexibility, interface defects, and traps caused by materials mismatches.

The goal of this work is to design a mechanically stacked MJ solar cell model using Si and Ge and to demonstrate a viable manufacturing process for highly efficient photovoltaic (PV) devices on single crystal substrates (epitaxial substrates) in the shape of vertically oriented micro/nano-scale 1D pillars and 2D walls and transfer them to inexpensive, lightweight, and amorphous surfaces using a mass-manufacturable fracture-transfer-print tool developed at UC Davis[12]. In the study, the transmittance, reflectance and absorbance of $\mathrm{Si}$, and $\mathrm{Ge}$ 1D nano-pillar and 2D nano-wall arrays have been calculated numerically to compare with the conventional planar structures. By introducing 1D and 2D nanostructures to solar cells a high carrier collection efficiency and enhancement in absorption are calculated owing to better transportation ability and light trapping property of 1D and 2D nanostructures.

\section{DEVICE MODEL}

In the proposed fracture-transfer print method, the original wafers will be repeatedly used for generating more devices during this PV manufacturing process. This will greatly decrease the cost of PVs by dramatically reducing the material consumption and making it environmentally benign while offering high efficiency and flexibility of panels for ease of packaging and installations. Fracture-transfer-print technology as explained in Fig.1 offers a new generation of heterogeneously integrated high-performance solar cells on lightweight and flexible substrates, exploiting the unique optical, mechanical and electrical properties of multiple semiconductor microstructures.

In our mechanically stacked MJ solar cell model, each subcell is designed on single crystalline substrates in the shape of vertically oriented micro/nano-scale $1 \mathrm{D}$ pillars and $2 \mathrm{D}$ walls and subsequently transfers them to inexpensive, light weight, and amorphous surfaces using the fracture-transfer-print tool.

In the simulations, the modeled mechanically stacked MJ solar cell is chosen to consist of two-junction cells, made of $\mathrm{Si}$, and Ge materials as top and bottom subcells, respectively. The stack structure is shown in Figure 2(b).

Such a design allows the extraction of power separately from each subcell, which is not the case in monolithically stacked MJ solar cell. Silicon was chosen as a top subcell in our proposed mechanically stacked MJ solar cell. With band gap energy value around $1.1 \mathrm{eV}, \mathrm{Si}$ is ideally suited for the bottom junction. It is also well-known that compared with other materials Si substrates are more convenient and cheap for the solar cells. 


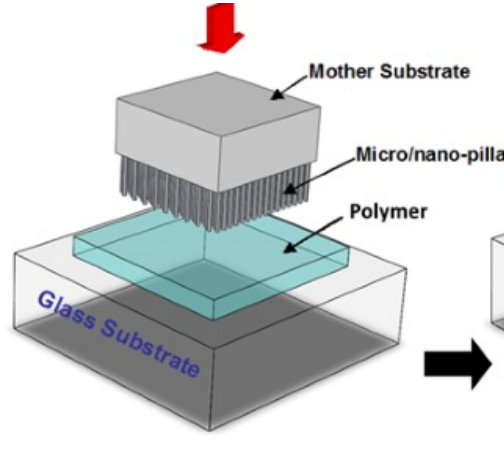

(a) Vertical Embossing

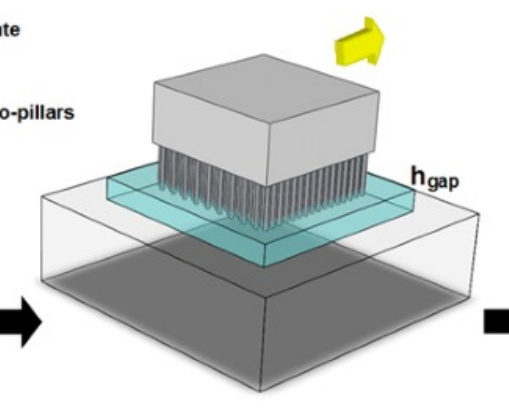

(b) Lateral Fracturing

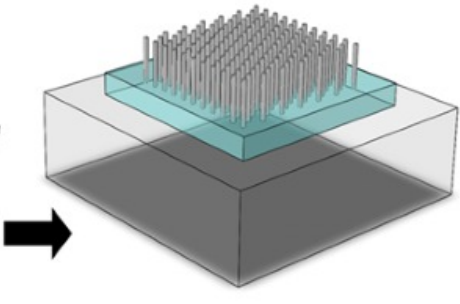

(c) Transferred Pillars

Figure 1. Explanation of the fracture-transfer print technique; (a) Vertical embossing of the mother substrate into the polymer substrate by applying a uniform vertical force, (b) lateral fracturing of the 1D pillars or 2D walls, (c) transferred micro/nano structres into the polymer layer.

For the bottom cell in our mechanically stacked MJ solar cell the Ge has been chosen. It is an excellent bottom junction material in a MJ solar cell[1]. Ge has a direct band gap nature with energy value of around $0.67 \mathrm{eV}$. It has wider wavelength of optical absorption of solar spectrum compared to the Si covers. That is, as shown in Figure 2(a), Ge is covering $300 \mathrm{~nm}-1600 \mathrm{~nm}$ spectral range, while it is $300 \mathrm{~nm}-1060 \mathrm{~nm}$ for $\mathrm{Si}$. The band gap of $\mathrm{Si} / \mathrm{Ge}$ combination is very close to the optimum band gap arrangement for MJ solar cell to give the maximum efficiency.

a)

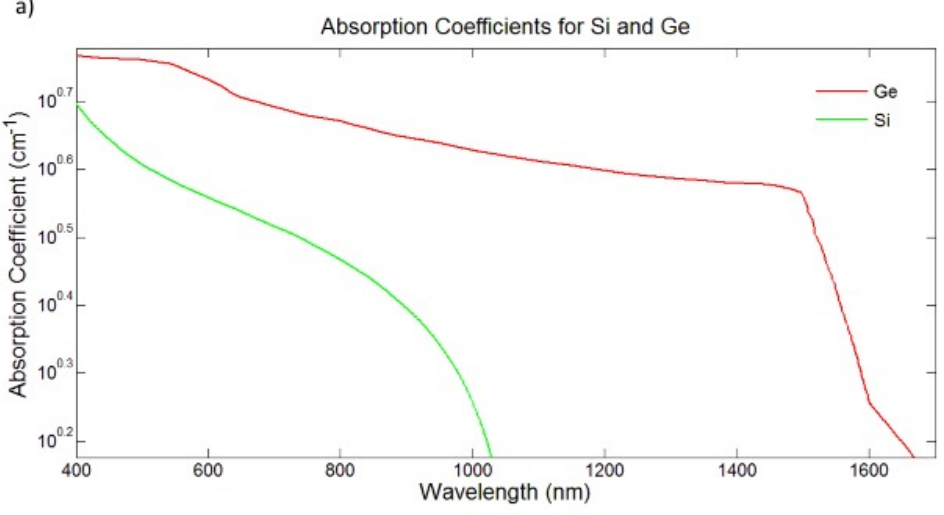

b)

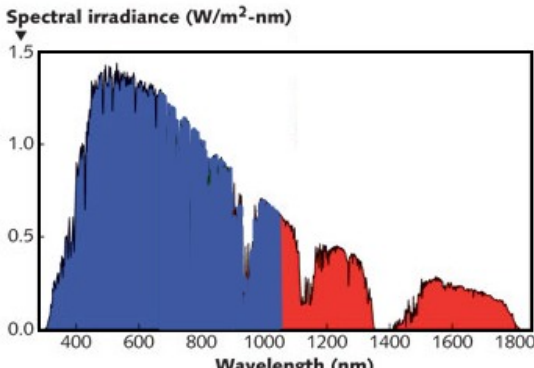

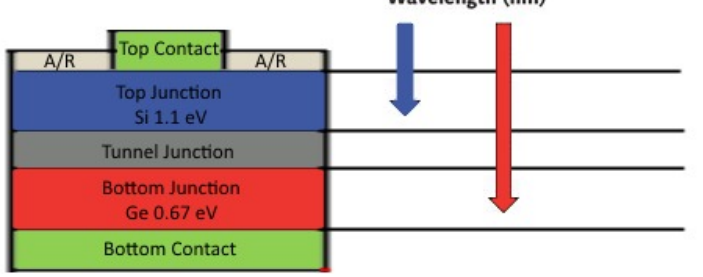

Figure 2. (a) Absorption coefficient graphs of Si (green) and Ge (red) materials, (b) Proposed stacked solar cell structure as $\mathrm{Si}$ being the top junction and Ge being the bottom junction. Absorption regions of the materials in the solar spectrum is shown for $\mathrm{Si}$ (blue) and $\mathrm{Ge}$ (red).

Figure 3 shows the 3D view of our proposed mechanically stacked MJ solar cell, consisted of 1D nanopillas and 2D nanowall structured $\mathrm{Si}$ and Ge layers as top and bottom subcells, respectively. Four different structures are proposed that can be used by the fracture-transfer-print technique; (a) axial junction 2D micro/nano walls, (b) axial junction 1D micro/nano pillars. It's assumed that after the top and bottom cells are mechanically stacked as described in the literature[13], material etching techniques such as Deep Reactive Etching, could be used for the fabrication of the proposed structures. Fabrication of such structure with high aspect ratio is shown in the literature in detail. Our theoretical calculations showed that the successfully transfer of these structures with the length up to $30 \mu \mathrm{m}$ is possible by using the fracture-transfer print technique. Therefore, in the simulations we limited the total device thickness up to this length. 

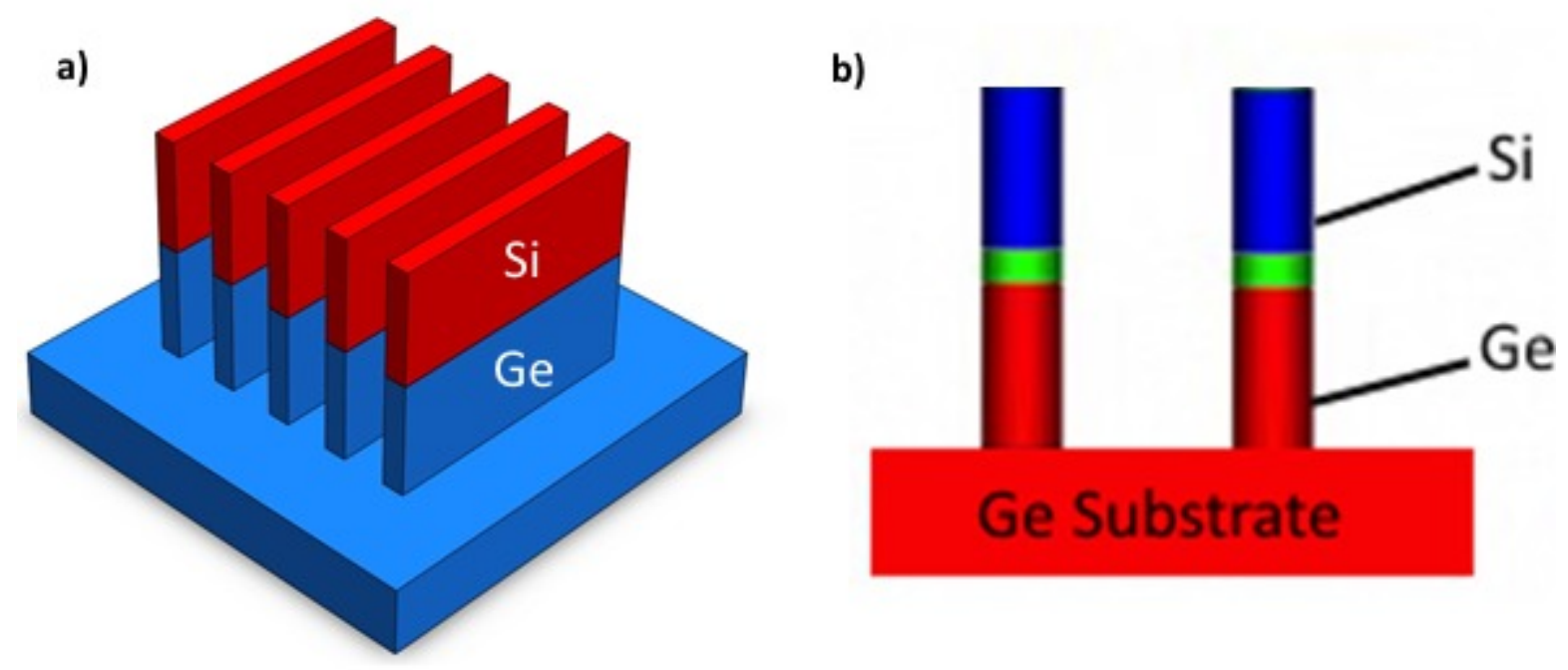

Figure 3. Proposed 2D wall (a) and 1D pillar (b) multistacked solar cell structures with having Si solar cell as the top cell and Ge solar cell as the bottom cell.

\section{RESULTS AND DISCUSSION}

Proposed 2D walls and 1D pillars of the multistacked microstructured solar cells are studied analytically in terms of their optical characteristics and device performance. For the optical characterization, Absorption and reflection spectra analysis are studied based on the effective medium theory[14]. According to this theory, to calculate the absorption and reflection spectra of the Si and Ge thin films, equation (1) to (3) are used;

$$
\begin{gathered}
A=1-R-T \\
R=\left|\frac{1}{2}\left(n_{e}-\frac{1}{n_{e}}\right) \sin \left(n_{e} k L\right)\right|^{2} T \\
T=\left|\cos \left(n_{e} k L\right)-\frac{i}{2}\left(n_{e}+\frac{1}{n_{e}}\right) \sin \left(n_{e} k L\right)\right|^{-2}
\end{gathered}
$$

where $\mathrm{L}$ is the thickness of the thin film, $\mathrm{k}$ is the wavenumber of the light and $n_{e}$ is the effective refractive index. In the analysis, $\mathrm{L}$ is chosen as $100 \mu \mathrm{m}$ and $\mathrm{k}$ and $n_{e}$ are taken from the reported results in the literature. As seen in figure 4(a), when the fill ratio is 1, absorption of Si solar cell is around 0.55 in the range of $400 \mathrm{~nm}$ to $550 \mathrm{~nm}$ and decreases as the wavelength increases. As the fill ratio decreases, the wavelength range for the high absorption decreases. It is interesting to note that highest absorption values are achieved as 0.6 in the range of $400 \mathrm{~nm}$ to $450 \mathrm{~nm}$ when the fill ratio is around 0.9 . Similarly, according to figure 4 (b), when the fill ratio is 1 , absorption of Ge is 0.5 in the wavelength range of 400 $\mathrm{nm}$ to $1000 \mathrm{~nm}$ and starts to decrease as the wavelength increases. Highest absorption values for Ge solar cell can be found as 0.8 when the fill ratio of the device is between 0.43 and 0.78 .

The solar cell optimization of each subcell was carried out based on previously reported experimentally or theoretically calculated parameters of $\mathrm{Si}$, and $\mathrm{Ge}$ materials as given in Table $1[15,16]$. 

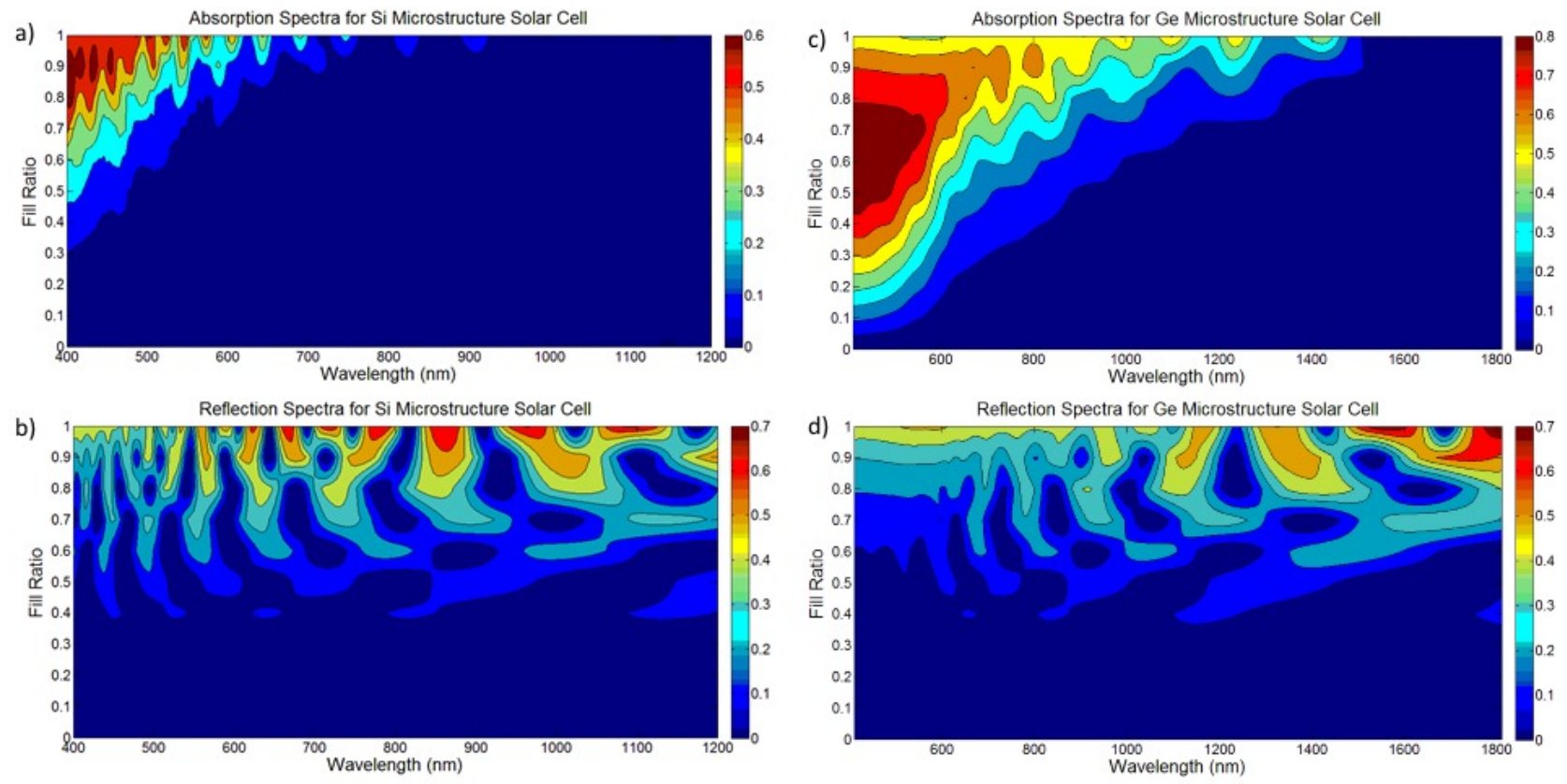

Figure 4. Absorption and Reflection specta of Si solar cell (a) and (b) and Ge solar cell (c) and (d) are given respectively.

Table 1. Device parameters of $\mathrm{Si} / \mathrm{Ge}$ mechanically stacked inorganic solar cell model.

\begin{tabular}{lc|c|c}
\multicolumn{2}{c|}{ Types and Definitions } & Si top cell & Ge bottom cell \\
\hline $\mathrm{E}_{\mathrm{g}}(\mathrm{eV})$ & (bandgap) & 1.124 & 0.67 \\
\hline $\mathrm{N}_{\mathrm{A}}\left(\mathrm{cm}^{-3}\right)$ & (acceptor density) & $1 \times 10^{20}$ & $1 \times 10^{19}$ \\
\hline $\mathrm{N}_{\mathrm{D}}\left(\mathrm{cm}^{-3}\right)$ & (donor density) & $1 \times 10^{15}$ & $1 \times 10^{17}$ \\
\hline $\mathrm{n}_{\mathrm{i}}\left(\mathrm{cm}^{-3}\right)$ & (intrinsic carrier concentration) & $1 \times 10^{10}$ & $2.4 \times 10^{13}$ \\
\hline $\mathrm{D}_{\mathrm{A}}\left(\mathrm{m}^{2} / \mathrm{s}\right)$ & (minority carrier diffusion constant) & 12 & 46.8 \\
\hline $\mathrm{D}_{\mathrm{N}}\left(\mathrm{m}^{2} / \mathrm{s}\right)$ & (minority carrier diffusion constant) & 36 & 101.4 \\
\hline$\tau_{\mathrm{e}}(\mu \mathrm{s})$ & (minority carrier lifetime) & 80 & $1 \times 10^{-3}$ \\
\hline$\tau_{\mathrm{h}}(\mu \mathrm{s})$ & (minority carrier lifetimes) & $7 \times 10^{-4}$ & $1 \times 10^{-3}$ \\
\hline
\end{tabular}

The current density-voltage (J-V) characteristics of Si and Ge subcells are calculated according to equation (4) where the saturation current density, $J_{0}$, is defined in equation (5) and light generated current density, $J_{L}$, is defined in equation (6). In these equations, $L_{e}$ and $L_{h}$ are the electron and hole minority carrier diffusion length respectively, $d$ is the device thickness, $T$ is the device thickness, $k_{B}$ is the Boltzmann's constant, $q$ is the elementary charge, $P F(\lambda)$ is the photon flux at the wavelength of $\lambda, \alpha(\lambda)$ is the absorption coefficient of material at the wavelength of $\lambda$. In the equation (4), diode ideality is chosen as 1 with no series and shunt resistance. The power conversion efficiencies of these subcells are calculated for different subcell thicknesses as shown in Figure 5. Details of this calculations are given elsewhere[17].

$$
\begin{aligned}
& J(V)=J_{0}\left(e^{q V / k_{B} T}-1\right)-J_{L} \\
& J_{0}=q n_{i}^{2}\left(\frac{D_{e}}{N_{A} L_{e}}+\frac{D_{h}}{N_{D} L_{h}}\right)
\end{aligned}
$$




$$
J_{L}=\int_{\lambda_{0}=280}^{\lambda_{1}=2000} q P F(\lambda)\left(1-e^{-\alpha(\lambda) d}\right) \Delta \lambda
$$

In these analysis, Si top cell thickness s considered between 0 and $20 \mu \mathrm{m}$ whereas Ge bottom cell thickness is chosen between 0 and $10 \mu \mathrm{m}$. As shown in Figure 5, overall device efficiency is computed be adding the individual subcell efficiencies directly.

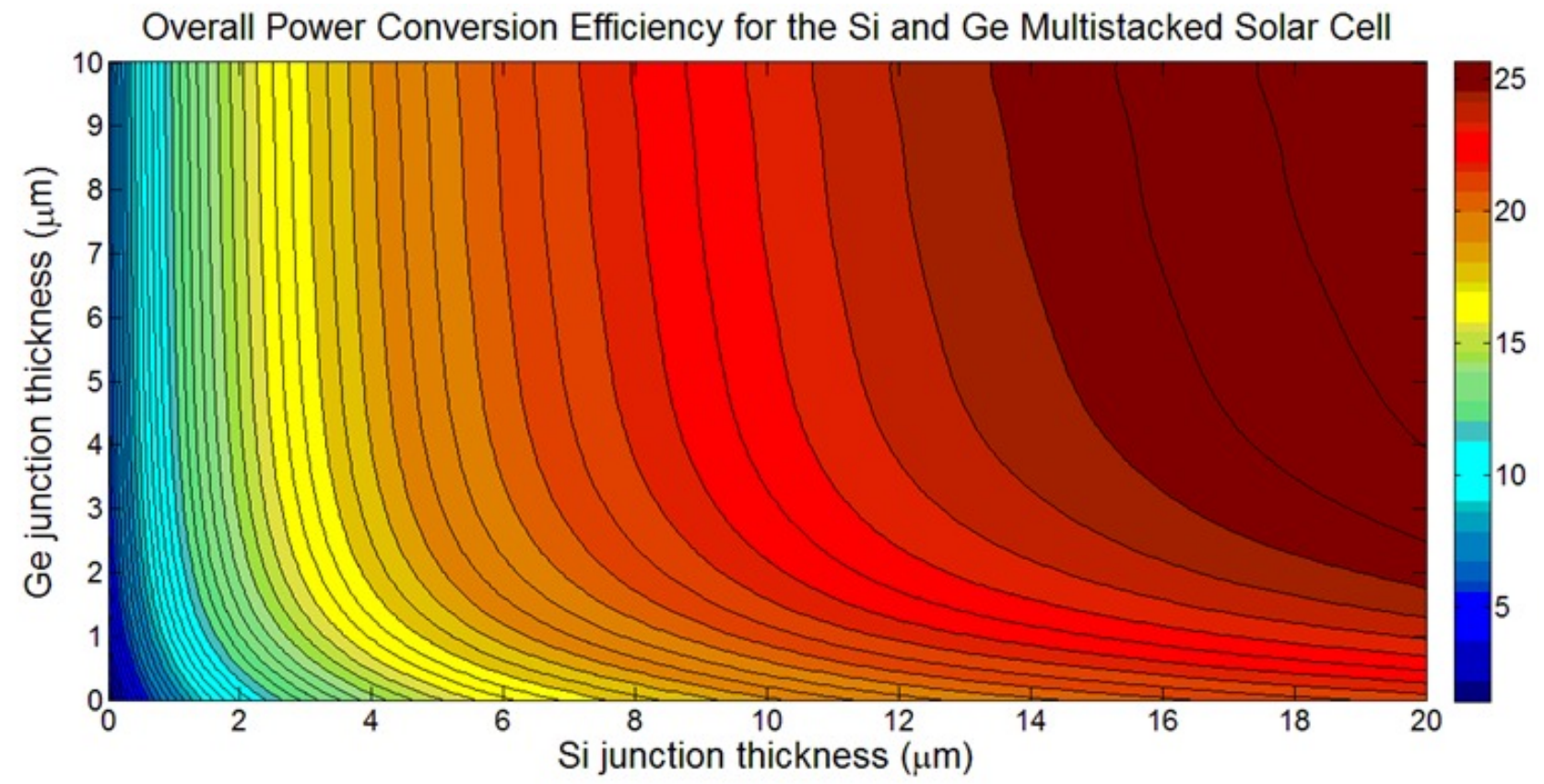

Figure 5. Calculated limits of the Power Conversion Efficiency for the Si/Ge mechanically stacked solar cells for the top Si junction thickness varying between $0 \mu \mathrm{m}-20 \mu \mathrm{m}$ and the bottom Ge junction thickness varying between $0 \mu \mathrm{m}-10 \mu \mathrm{m}$ at $\mathrm{T}$ $=300 \mathrm{~K}, 1000 \mathrm{~W} / \mathrm{m}^{2}$, AM1.5G.

According to Figure 5, to reach an efficiency of \%26, minimum device thickness must be chosen as $30 \mu \mathrm{m}$ by adjusting the Si top cell thickness to $20 \mu \mathrm{m}$ and Ge bottom cell thickness to $10 \mu \mathrm{m}$. Although increasing the thickness of the combined device, transfer yield of these structures decreases.

\section{CONCLUSION}

In this study, power conversion efficiencies of multistacked $\mathrm{Si} / \mathrm{Ge}$ solar cells are found to saturate at $\% 26$ with a combined device thickness of $30 \mu \mathrm{m}$ which is suitable for the proposed mass-manufacturable fracture-transfer-print technique. Also according to the simulation results, introducing microstructures such as $1 \mathrm{D}$ pillars or 2D walls into the $\mathrm{Si}$ and Ge single junction devices, increases the absorption which is related with the power conversion efficiency. The results show that multistacked 1-D and 2-D microstructured $\mathrm{Si} / \mathrm{Ge}$ solar cells are promising candidates to overcome the Si practical efficiency limit of $26 \%$ by using less than $\% 10$ of the mother substrate.. 


\section{REFERENCES}

[1] D. R. L. H. L. Cotal, J. H. Ermer, R. R. King, N. H. Karam, S. R. Kurtz, D. J. Friedman, J. M. Olson, S. Ward, A. Duda, K. A. Emery, T. Moriarty, [Highly Efficient 32.3\% Monolithic GaInP/GaAs/Ge Triple Junction Concentrator Solar Cells ], (2000).

[2] J. P. Bailbe, A. Val, A. Marty et al., "Techniques for epitaxial lift-off - Applications and future outlook," Journal De Physique Iv, 9(P2), 113-122 (1999).

[3] R. Basco, A. Prabhu, K. S. Yngvesson et al., "Monolithic integration of a $94 \mathrm{GHz}$ AlGaAs/GaAs 2-DEG mixer on quartz substrate by epitaxial lift-off," Ieee Transactions on Electron Devices, 44(1), 11-16 (1997).

[4] K. Furukawa, and A. Nakagawa, "Applications of the Silicon-Wafer Direct-Bonding Technique to Electron Devices," Applied Surface Science, 41-2, 627-632 (1989).

[5] J. Ohura, T. Tsukakoshi, K. Fukuda et al., "A Dielectrically Isolated Photodiode Array by Silicon-Wafer Direct Bonding," Ieee Electron Device Letters, 8(10), 454-456 (1987).

[6] G. Roelkens, J. Van Campenhout, J. Brouckaert et al., "III-V/Si photonics by die to wafer bonding," Materials Today, 10(7-8), 36-43 (2007).

[7] V. J. Logeeswaran, A. Sarkar, M. S. Islam et al., "A 14-ps full width at half maximum high-speed photoconductor fabricated with intersecting InP nanowires on an amorphous surface," Applied Physics aMaterials Science \& Processing, 91(1), 1-5 (2008).

[8] J. Geske, Y. L. Okuno, J. E. Bowers et al., "Vertical and lateral heterogeneous integration," Applied Physics Letters, 79(12), 1760-1762 (2001).

[9] A. Sarkar, I. Kimukin, C. W. Edgar et al., "Heteroepitaxial growth dynamics of InP nanowires on silicon," Journal of Nanophotonics, 2, (2008).

[10] E. G. Bauer, B. W. Dodson, D. J. Ehrlich et al., "Fundamental Issues in Heteroepitaxy - a Department-ofEnergy, Council-on-Materials-Science Panel Report," Journal of Materials Research, 5(4), 852-894 (1990).

[11] Y. B. Bolkhovityanov, O. P. Pchelyakov, L. V. Sokolov et al., "Artificial GeSi substrates for heteroepitaxy: Achievements and problems," Semiconductors, 37(5), 493-518 (2003).

[12] V. J. Logeeswaran, A. M. Katzenmeyer, and M. S. Islam, "Harvesting and Transferring Vertical Pillar Arrays of Single-Crystal Semiconductor Devices to Arbitrary Substrates," Ieee Transactions on Electron Devices, 57(8), 1856-1864 (2010).

[13] D. Kim, Y. Choi, E. C. Do et al., "High Efficiency Silicon and Germanium Stack Junction Solar Cells," 2012 Ieee International Electron Devices Meeting (Iedm), (2012).

[14] Z. Xiong, F. Zhao, J. Yang et al., "Comparison of optical absorption in Si nanowire and nanoporous Si structures for photovoltaic applications," Applied Physics Letters, 96(18), 181903 (2010).

[15] M. B. Prince, "Drift Mobilities in Semiconductors .1. Germanium," Physical Review, 92(3), 681-687 (1953).

[16] M. B. Prince, "Drift Mobilities in Semiconductors .2. Silicon," Physical Review, 93(6), 1204-1206 (1954).

[17] R. A. Janssen, and J. Nelson, "Factors limiting device efficiency in organic photovoltaics," Adv Mater, 25(13), 1847-58 (2013). 